\title{
Towards a dislocation-based model for strain path effects in bainitic pipeline steels
}

\author{
Matthias Bönisch, Marc Seefeldt, Albert Van Bael, Nuria Sanchez and Steven Cooreman
}

Matthias Bönisch. KU Leuven, Department of Materials Engineering, Kasteelpark Arenberg 44, box 2450, 3001 Leuven, Belgium Corresponding author: matthias.bonisch@kuleuven.be

Marc Seefeldt. KU Leuven, Department of Materials Engineering, Kasteelpark Arenberg 44, box 2450, 3001 Leuven, Belgium

Albert Van Bael. KU Leuven, Department of Materials Engineering, Kasteelpark Arenberg 44, box 2450, 3001 Leuven, Belgium

Nuria Sanchez. ArcelorMittal Global R\&D Gent, OCAS NV, Pres. J. F. Kennedylaan 3, 9060 Zelzate, Belgium

Steven Cooreman. ArcelorMittal Global R\&D Gent, OCAS NV, Pres. J. F. Kennedylaan 3, 9060 Zelzate, Belgium

\begin{abstract}
Modern pipeline steels exhibit complex microstructures that cause mechanical anisotropy in various respects. For instance, strain path effects under non-monotonic loadings are exceptionally pronounced in these steels. Crystallographic texture and morphological anisotropy are the main contributors to strength and hardening directionality in pipeline steels under monotonic loading. In contrast, the dislocation substructure is seen as the primary source for Bauschinger and cross effects during complex non-monotonic loading, e.g. during pipe forming. The Bauschinger effect for example may arise from pile-ups formed at obstacles such as intragranular shear bands, and homo- or heterophase boundaries. The dislocation-based model by Peeters et al. [Acta Mater., 49 (2001), pp. 1607-1619] developed for coarse-grained ferritic steel allows for complex strain path effects through the accumulation of dislocations at micro-shear bands. However, it struggles to reproduce the large Bauschinger effect of $\sim 250 \mathrm{MPa}$ in fine-grained bainitic pipeline steel [Bönisch et al., Procedia Manuf., 47 (2020), pp. 1434-1441]. Considering the microstructural differences between the two steel varieties, a promising way to improve the model predictions - especially for the Bauschinger effect - is to incorporate dislocation interactions with phase and/or grain boundaries. In the present work, we introduce this approach and demonstrate the basic capabilities of such a grain boundary-extended Peeters model. By accounting for the formation of pile-ups at grain boundaries the Bauschinger effect is enlarged. Furthermore, by explicitly considering the grain boundary spacing, the model can deliver grain size (Hall-Petch) strengthening.
\end{abstract}

Keywords. Bauschinger Effect, Bainitic Steel, Multiscale Modelling, Anisotropy, Grain Boundaries, Substructure

\section{Introduction}

Bainitic high-strength low-alloy (HSLA) steels are widely used for the construction of pipelines for long-distance liquid and gas transport. The ability to control and predict pipe mechanical behavior is crucial to ensure their safe operation. However, the pronounced mechanical anisotropy of hot-rolled plates combined with the alteration of the mechanical response by pipe forming can seriously compromise pipe reliability. Yet, predicting the mechanical response of pipeline steel represents a significant challenge. It is above all the exceptionally strong Bauschinger effect, manifesting itself in HSLA steels as a yield stress reduction on reverse loading of up to 50\% [1-3], that complicates the successful prediction of the pipe mechanical response.

Modern pipeline steels consist of different constituents, such as ferrite (polygonal, acicular, granular, bainitic), martensite-austenite, and non-metallic inclusions [2, 4-7]. These complex microstructures abound with obstacles, such as intragranular shear bands, homo- or heterophase boundaries, which in turn impede dislocation motion, leading to pile-up formation. The relatively easy release of dislocations from those pile-ups is generally considered as the main source of the Bauschinger effect upon load reversal.

Recent numerical studies have shown that state-of-the-art plasticity models have difficulties to accurately predict anisotropy evolution during pipe forming $[1,3]$. This shortcoming applies to both advanced phenomenological models 
Towards a dislocation-based model for strain path effects in bainitic pipeline steels

and dislocation-based models inspired by the intragranular substructure. Our previous work using the dislocationbased substructural hardening model by Peeters et al. [8] suggested that a faithful prediction of property evolution of bainitic pipeline steel in response to load path changes as they occur during pipe forming can be largely improved by accounting for ferrite interfaces [3].

The challenges lying ahead are two-fold: first, an accurate micro-scale hardening law is required. Dislocation-based models which explicitly include the dislocation substructure and pile-up formation are favored, since substructure and pile-ups are the major sources of the Bauschinger effect. This law is still to be developed and, based on our experience, should incorporate intragranular shear bands and grain boundaries as directional, planar glide obstacles including their crystallographic alignments. Second, once found, this novel law is to be embedded into a multi-scale simulation framework to address the materials' response to strain path changes for macroscopic simulations of pipe forming.

\subsection{Dislocation-based hardening models for cubic materials}

In the last 2 decades, several crystal plasticity-based models have been proposed for simulating the hardening response of plastic polycrystals subjected to simple or complex strain paths.

In line with the early derivations of Hall-Petch type strength and hardening contributions $[9,10]$, the model by Sinclair et al. [11] explicitly distinguished two hardening contributions due to dislocations accumulating at grain boundaries: (i) Taylor-type hardening due to the stored additional dislocation density and (ii) back stresses linked to specific (nonscreened) configurations of excess dislocations. In contrast to strain gradient plasticity theories, this approach not only lifted the ambiguity of hardening due to "geometrically necessary dislocations" but also recognized the crucial role of their spatial arrangement.

An alternative continuum model was put forward by Rauch et al. based on the observation of massive dislocation annihilation in $\mathrm{Al}$ alloys and low carbon steels by reverse straining [12,13]. The first model version divided the global dislocation population into two different types, labelled forward and reverse. The former type becomes activated by reverse shear and allows simulating work hardening for the special case of a reversed proportional strain path [12]. Later, this model was extended to cross-loading by introducing a third dislocation density and a parameter which measures the amplitude of the strain path change [13]. Kitayama et al. [14] reformulated that model within a crystallographic framework to incorporate slip directionality and texture effects. Instead of considering global dislocation densities, each slip system was associated with forward and reverse dislocations to allow for more general strain path changes. A similar, crystallographically-resolved approach was recently demonstrated by Bong et al. [15] for ferritic steel. In contrast, Carvalho Resende et al. [16] combined the earlier Rauch models with a grain-size dependent tensorial back-stress expression. In this way, they described plastic anisotropy and Bauschinger effect in IF steel for constant crystallographic texture.

When dislocations are not impeded by precipitates nor by grain boundaries, an intragranular dislocation substructure develops in bcc single-phase metals and alloys [17-19]. That substructure consists of randomly oriented cells and crystallographically oriented cell block boundaries (CBBs). In the model by Peeters et al. [8, 20], the substructural features in bcc materials are associated to three dislocation densities: $\rho$ (cells), $\rho_{i}{ }^{w d}$ (CBB on the $i$-th $\{110\}$ plane) and $\rho_{i}{ }^{w p}$ (net dislocation pile-up at CBB $i$ ). While the pile-ups polarize the CBBs and cause reverse (Bauschinger) effects upon strain reversal, the CBBs give rise to latent hardening and cross-effects. Each slip system exhibits thus a different critical shear stress, which furthermore depends on the shear direction. In addition, the model considers that upon reloading, the previously developed cell substructure disintegrates, leading to a plateau on the stress-strain curve (hardening-stagnation). Integrated into the relaxed-constraint grain-interaction crystal plasticity framework ALAMEL [21], the Peeters model has successfully predicted the stress evolution during complex strain path changes and drawing operations for coarse-grained IF steels [22]. 


\subsection{Present work}

Despite the success of Peeters' model, our recent work suggested that incorporation of dislocation interactions with phase and/or grain boundaries are important to reliably reproduce the large Bauschinger effect exhibited by X70 pipeline steels [3]. Dislocation-dislocation interactions alone could not explain the strong stress differentials upon load reversal. Advanced bainitic steels such as X70 exhibit fine microstructures with a ferrite grain size, the main constituent, of 1-15 $\mu \mathrm{m}$. In contrast to the coarse-grained IF steel for which the Peeters model was originally developed, $\mathrm{X} 70$ steel is thus very rich in grain boundaries. In the present work, we develop equations that extend the original Peeters model to dislocation-grain boundary interactions. These interactions contribute to grain-size strengthening (Hall-Petch effect) as well as to the Bauschinger effect by the formation of dislocation pile-ups at the grain boundaries. The present work is thus an important step towards an improved material model for fine-grained bcc materials.

\section{Model development}

\subsection{Incorporating grain boundaries into the Peeters model}

The model developed here schematizes the central features of the grain-boundary and dislocation substructure as illustrated in Fig. 1. Relative to Peeters' model it adds (i) straight grain boundary segments and (ii) for each grain boundary segment a new dislocation density $\rho_{j}^{g p} \cdot \rho_{j}^{g p}$ represents dislocations piling up at a grain boundary and polarizing it.

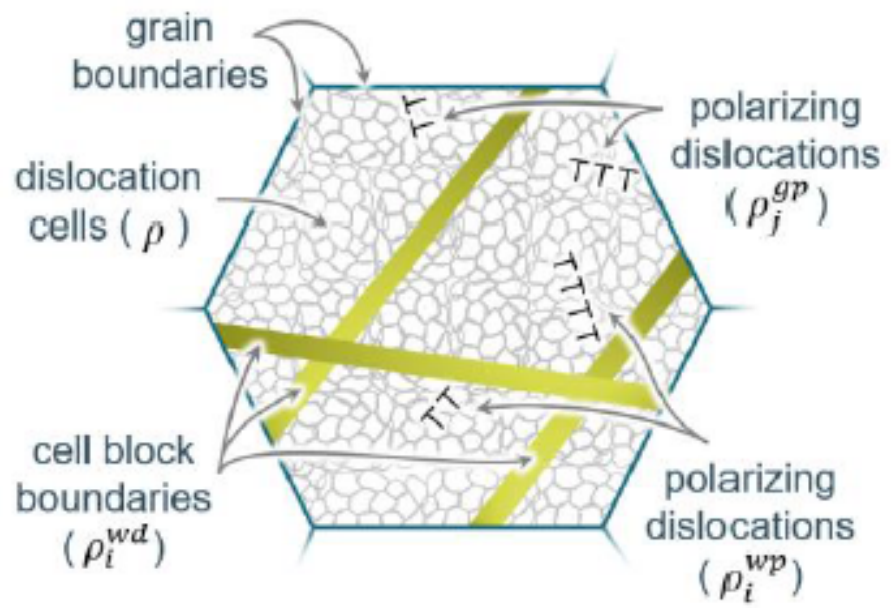

Fig. 1: Schematic representation of the grain-boundary and dislocation substructure

In the following description we focus on the grain-boundary related features, while the remaining features are described in $[8,20]$. Grain boundary pile-ups form by dislocation interaction with the grain boundaries and with other dislocations already accumulated there. On the other hand, interaction between dislocations accumulating on different slip systems may cause the destruction and screening of pile-ups. The following equation thus describes the accumulation of $\rho_{j}^{g p}$ : 
Towards a dislocation-based model for strain path effects in bainitic pipeline steels

$$
\dot{\rho}_{j}^{g p}=\left[\operatorname{sign}\left(\Phi_{j}^{g p}\right)\left(I_{\rho}^{g p} \sqrt{\left|\rho_{j}^{g p}\right|}+\frac{I_{d}^{g p}}{d_{j}^{g}}\right)-R^{g p} \rho_{j}^{g p}\right]\left|\Phi_{j}^{g p}\right|
$$

Note that $\rho_{j}{ }^{g p}$ can be $>0$ or $<0$ due to $\Phi_{j}^{g p}$, the net flux of dislocations onto the grain boundary:

$$
\Phi_{j}^{g p}=\sum_{s=1}^{n} \frac{\dot{\gamma}_{s}}{b} \boldsymbol{s}_{s}^{b} \cdot \boldsymbol{n}_{j}^{g}
$$

with $n$ the number of slip systems, $\dot{\gamma}_{s}$ and $\boldsymbol{s}_{S}{ }^{b}$ the shear rate and slip direction for slip system $s, b$ the magnitude of the Burgers vector, and $\boldsymbol{n}_{j}{ }^{g}$ the grain boundary normal vector. The scalar product in (2) takes into account that the storage probability depends on the relative orientation of the incoming dislocation and the grain boundary. $I_{\rho}^{g p}$ and $I_{d}{ }^{g p}$ are the storage parameters due to dislocation-dislocation and due to dislocation-grain-boundary interactions, respectively. $d_{j}{ }^{g}$ is the distance between 2 grain boundary segments of orientation $j . R^{g p}$ characterizes the dynamic recovery in line with the Kocks-Mecking approach [23]. When the net flux for a grain boundary is reversed,

$$
\dot{\rho}_{j}^{g p}=-R_{\text {rev }} \rho_{j}^{g p}\left|\Phi_{j}^{g p}\right|
$$

until $\rho_{j}{ }^{g p}$ changes sign. A change of sign of $\rho_{j}^{g p}$ indicates that the net pile-up now forms on the other side of the grain boundary. The homogenized current critical resolved shear stress (CRSS) on slip system $s$ is obtained by weighted summation of all relevant flow stress contributions:

$$
\tau_{s}^{c}=\tau_{0}+(1-f) \tau^{C B}+f \sum_{i}^{6}\left(\tau_{i s}^{w p}+\tau_{i s}^{w d}\right)+\sum_{j}^{G B \text { segments }}\left(\tau_{j s}^{g p}+\tau_{j s}^{g}\right)
$$

$\tau_{0}$ is the Peierls stress, $f$ the volume fraction of CBBs, $\tau^{C B}$ the isotropic flow stress contribution from dislocation cells; $\tau_{i s}{ }^{w p}$ represents the back-stress from pile-ups at CBB $i$, and $\tau_{i s}{ }^{w d}$ is the contribution of the corresponding CBB itself. For each grain boundary segment, the second sum in (4) gathers the back-stress

$$
\left.\tau_{j s}^{g p}=\alpha G b \mid \sqrt{\left|\rho_{j}^{g p}\right|} \boldsymbol{s}_{s}^{b} \cdot \boldsymbol{n}_{j}^{g} \operatorname{sign}\left(\rho_{j}^{g p}\right)\right)
$$

and the grain-size strengthening 


$$
\tau_{j s}^{g}=\left|\boldsymbol{s}_{s}^{b} \cdot \boldsymbol{n}_{j}^{g}\right| \frac{k_{g}}{\sqrt{d_{j}^{g}}}
$$

with $\alpha$ characterizing the dislocation interaction strength, $G$ the shear modulus, and $k_{g}$ the micro-scale Hall-Petch parameter. $\langle\ldots\rangle$ in (4) indicates the Heaviside function

$$
\langle x\rangle=\left\{\begin{array}{lll}
x & \forall x \geq 0 \\
0 & \forall x<0
\end{array}\right.
$$

which lets dislocations escape from pile-ups without hindrance when the shear direction is reversed. This results in a lower flow stress and reduced hardening on reverse loading, i.e. a Bauschinger effect, as we will demonstrate in the next section.

\subsection{Proof-of-concept}

We now demonstrate that the equations introduced above in section 2.1 give rise to the desired stress-strain features including increased Bauschinger effect and grain size strengthening. For this purpose, we set up a hypothetical microstructure with one slip system $\boldsymbol{s}_{S}{ }^{b}$, one $\operatorname{CBB} \boldsymbol{n}_{i}{ }^{w}$ and one grain boundary $\boldsymbol{n}_{j}{ }^{g}$ :

$$
\begin{aligned}
\boldsymbol{s}_{s}^{b} & =1 / \sqrt{3}\left[\begin{array}{lll}
1 & 1 & 1
\end{array}\right] \\
\boldsymbol{n}_{i}^{w} & =1 / \sqrt{2}\left[\begin{array}{lll}
1 & 1 & 0
\end{array}\right] \\
\boldsymbol{n}_{j}^{g} & =1 / \sqrt{3}\left[\begin{array}{lll}
1 & 1 & 1
\end{array}\right]
\end{aligned}
$$

Table 1 lists the values for the newly introduced model parameters for grain boundary features. For the storage and recovery rates for pile-ups at CBBs $\left(I^{w p}\right.$ and $\left.R^{w p}\right)$ and the remaining model parameters the values in [8] are used here. Disintegration of the random cell structure on load reversal by dislocations emitted from pile-ups at CBBs and at grain boundaries is neglected in the present setting.

Table 1. Grain boundary related model parameters

\begin{tabular}{lllll}
\hline Parameter & $I_{d}^{g p}$ & $I_{\rho}^{g p}$ & $R^{g p}$ & $k_{g}$ \\
\hline Unit & - & - & $\mathrm{m}$ & $\mathrm{MPa} / \mathrm{m}^{0.5}$ \\
\hline Value & 1 & $I^{w p} \sqrt{f}$ & $R^{w p}$ & 0.24 \\
\hline
\end{tabular}

Fig. 2 shows the simulation results for forward shear of 0.15 followed by reverse shear for two different grain sizes, $d_{j}{ }^{g}=50 \mu \mathrm{m}$ and $d_{j}{ }^{g}=2 \mu \mathrm{m}$. The calculated dislocation densities for cells, CBBs and pile-ups at CBBs are unaffected by the reduction in grain size. In contrast, the density of grain boundary pile-ups increases by more than 6 times when the 
Towards a dislocation-based model for strain path effects in bainitic pipeline steels

grain boundary spacing decreases from $50 \mu \mathrm{m}$ to $2 \mu \mathrm{m}$. The evolution of the CRSS shows a two-fold effect due to the reduction in grain size: (i) due to the Hall-Petch effect the overall stress-level increases by about $150 \mathrm{MPa}$ and (ii) the stress differential due to reversal of the shear direction $(\gamma=0.15)$ is enlarged by 35 MPa. As clearly illustrated in Fig. 2(b) and 2(d), the origin for the enlarged Bauschinger effect is the larger flow stress contribution stemming from the polarizing dislocations piling up at grain boundaries in case of small grains.
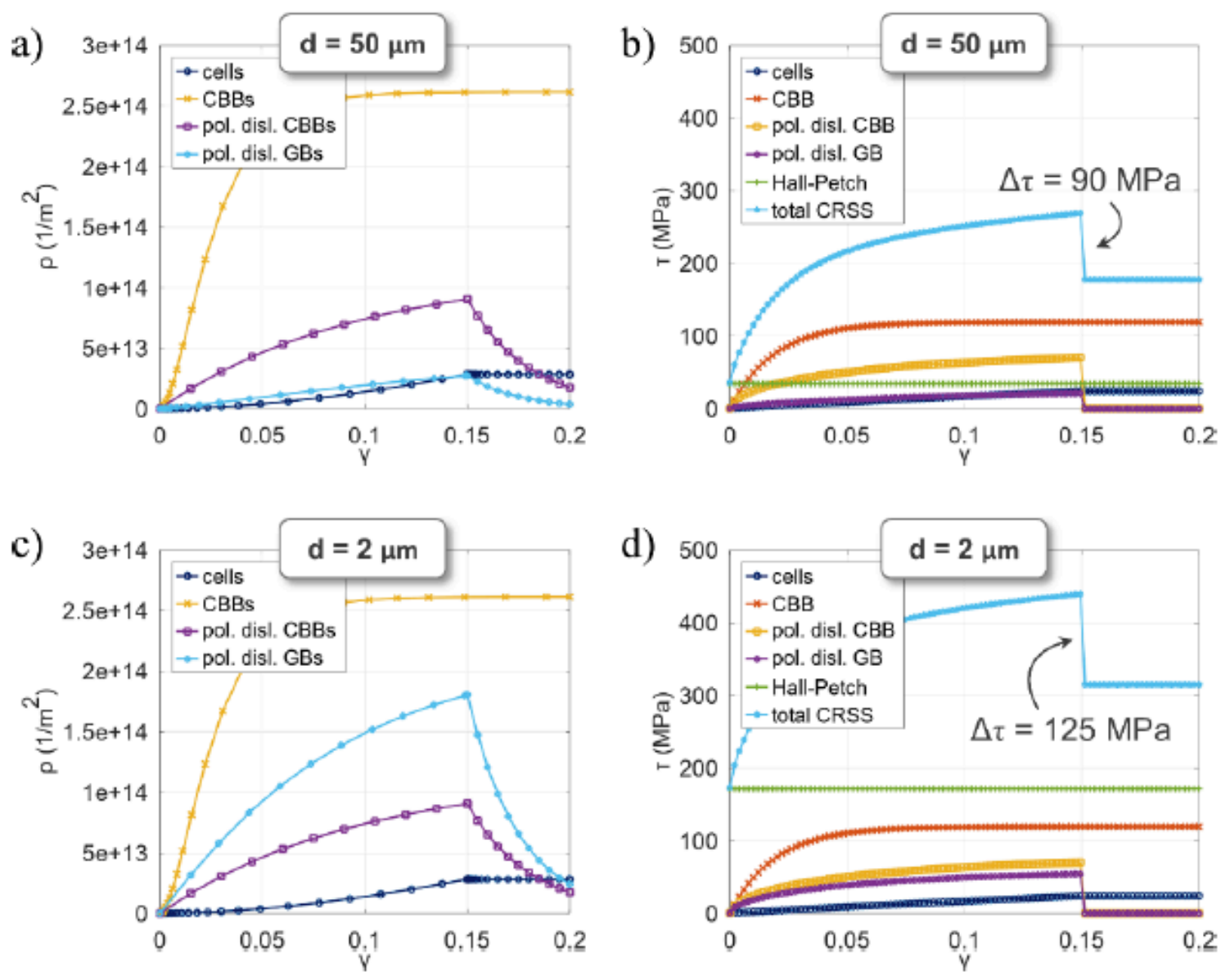

Fig. 2: Evolution of $(a, c)$ dislocation densities and of $(b, d)$ critical resolved shear stress as a function of total shear strain for forward shear followed by reverse shear. Two different grain sizes are illustrated

\section{Conclusions}

The presented work demonstrates the development of a novel dislocation-based crystal plasticity hardening model for fine-grained bainitic steel. By building on the well-established approach by Peeters et al. [8, 20] to schematize the dislocation substructure in bcc materials, a model is obtained that demonstrably incorporates anisotropy from dislocation interactions with both CBBs and grain boundaries. By explicitly including grain size into the evolution equation for grain boundary pile-ups, two desired effects are achieved: (i) grain size (Hall-Petch-type) strengthening and (ii) an enlarged stress differential on reverting the strain path corresponding to an increased Bauschinger effect. Our experience has shown that both are required to accurately predict the stress response during complex strain-path changes occurring during pipe forming of bainitic steel plates. With respect to the practical application of the suggested model, reliable calibration of the model parameters might be challenging. As a remedy, a sequential design strategy for 
global optimization of black-box functions, as demonstrated previously [3], might be considered.

\section{Acknowledgements}

The authors would like to thank Philip Eyckens for insightful discussions on the crystal plasticity source code.

\section{Bibliography}

[1] Cooreman, S. Van Hoecke, D. Liebeherr, M. Thibaux, P. Yamaguti Enderlin, M. Experimental and Numerical Study on the Evolution of Mechanical Properties During Spiral Pipe Forming, 11th Int. Pipeline Conf. (IPC2016). 2016. IPC2016-64183.

[2] Kim, DW. Sohn, SS. Kim, W-K. Kim, K-S. Lee, S. Study of Bauschinger effect of acicular ferrite and polygonal ferrite through ex-situ interrupted bending tests in API X80 linepipe steels. Scientific Reports, 2018, 8, 15598.

[3] Bönisch, M. Van Bael, A. Seefeldt, M. Barriobero-Vila, P. Requena, G. Sanchez, N. Cooreman, S. Unravelling Anisotropy Evolution during Spiral Pipe Forming: a Multiscale Approach. Procedia Manufacturing, 2020, 47, 1434-1441.

[4] Garcia, CI. Cho, K. Hua, M. DeArdo, AJ. The Alloy Design and Thermomechanically Controlled Processing (TMCP) of Plate for High Pressure, Large Diameter Pipelines, In: Thermec 2009, Editors: T. Chandra et al. 2010. 124-129.

[5] Carretero Olalla, V. Bliznuk, V. Sanchez, N. Thibaux, P. Kestens, LAI. Petrov, RH. Analysis of the strengthening mechanisms in pipeline steels as a function of the hot rolling parameters. Materials Science and Engineering: A, 2014, 604, 46-56.

[6] Sanchez Mouriño, N. Petrov, R. Bae, J-h. Kim, K. Kestens, LAI. Texture Dependent Mechanical Anisotropy of X80 Pipeline Steel. Advanced Engineering Materials, 2010, 12, 973-980.

[7] Omale, JI. Ohaeri, EG. Mostafijur, KM. Szpunar, JA. Fateh, F. Arafin, M. Through-Thickness Inhomogeneity of Texture, Microstructure, and Mechanical Properties After Rough and Finish Rolling Treatments in Hot-Rolled API 5L X70 Pipeline Steel. Journal of Materials Engineering and Performance, 2020, 29, 8130-8144.

[8] Peeters, B. Seefeldt, M. Teodosiu, C. Kalidindi, SR. Van Houtte, P. Aernoudt, E. Work-hardening/softening behaviour of b.c.c. polycrystals during changing strain paths: Part I. An integrated model based on substructure and texture evolution, and its prediction of the stress-strain behaviour of an IF steel during two-stage strain paths. Acta Materialia, $2001,49,1607-1619$

[9] Hall, EO. The Deformation and Ageing of Mild Steel: III Discussion of Results. Proceedings of the Physical Society Section B, 1951, 64, 747-753.

[10] Petch, NJ. The Cleavage Strength of Polycrystals. Journal of the Iron and Steel Institute, 1953, 174, 25-28.

[11] Sinclair, CW. Poole, WJ. Bréchet, Y. A model for the grain size dependent work hardening of copper. Scripta Materialia, 2006, 55, 739-742.

[12] Rauch, EF. Gracio, JJ. Barlat, F. Work-hardening model for polycrystalline metals under strain reversal at large strains. Acta Materialia, 2007, 55, 2939-2948.

[13] Rauch, EF. Gracio, JJ. Barlat, F. Vincze, G. Modelling the plastic behaviour of metals under complex loading conditions. Modelling and Simulation in Materials Science and Engineering, 2011, 19, 035009. 
Towards a dislocation-based model for strain path effects in bainitic pipeline steels

[14] Kitayama, K. Tomé, CN. Rauch, EF. Gracio, JJ. Barlat, F. A crystallographic dislocation model for describing hardening of polycrystals during strain path changes. Application to low carbon steels. International Journal of Plasticity, 2013, 46, 54-69.

[15] Bong, HJ. Lee, J. Lee, MG. Kim, D. Identification of mechanical responses of steel sheets under non-proportional loadings using dislocation-density based crystal plasticity model. International Journal of Mechanical Sciences, 2019, $155,461-474$.

[16] Carvalho Resende, T. Bouvier, S. Abed-Meraim, F. Balan, T. Sablin, SS. Dislocation-based model for the prediction of the behavior of b.c.c. materials - Grain size and strain path effects. International Journal of Plasticity, 2013, 47, 29-48.

[17] Peeters, B. Bacroix, B. Teodosiu, C. Van Houtte, P. Aernoudt, E. Work-hardening/softening behaviour of b.c.c. polycrystals during changing strain: Part II. TEM observations of dislocation sheets in an IF steel during two-stage strain paths and their representation in terms of dislocation densities. Acta Materialia, 2001, 49, 1621-1632.

[18] Šesták, B. Seeger, A. The Relationship between the Work-Hardening of B.C.C. and F.C.C. Metals. physica status solidi (b), 1971, 43, 433-444.

[19] Vincze, G. Rauch, EF. Gracio, JJ. Barlat, F. Lopes, AB. A comparison of the mechanical behaviour of an AA1050 and a low carbon steel deformed upon strain reversal. Acta Materialia, 2005, 53, 1005-1013.

[20] Peeters, B. Kalidindi, SR. Van Houtte, P. Aernoudt, E. A crystal plasticity based work-hardening/softening model for b.c.c. metals under changing strain paths. Acta Materialia, 2000, 48, 2123-2133.

[21] Van Houtte, P. Li, S. Seefeldt, M. Delannay, L. Deformation texture prediction: from the Taylor model to the advanced Lamel model. International Journal of Plasticity, 2005, 21, 589-624.

[22] Gawad, J. Eyckens, P. Roose, D. Van Bael, A. Van Houtte, P. Microstructure-informed simulation of strain reversal testing setup for sheet metal forming, 8th Forming Technology Forum - Advanced constitutive models in sheet metal forming. 2015. 129 - 134.

[23] Kocks, UF. Mecking, H. Physics and phenomenology of strain hardening: the FCC case. Progress in Materials Science, $2003,48,171-273$.

PDF automatically generated on 2021-05-20 08:21:18

Article url: https://popups.uliege.be/esaform21/index.php?id=2403

published by ULiège Library in Open Access under the terms and conditions of the CC-BY License

(https://creativecommons.org/licenses/by/4.0) 